\title{
Hybride Tensidsysteme mit anorganischen Bestandteilen
}

\author{
Sebastian Polarz,* Steve Landsmann und Alexander Klaiber
}

\begin{abstract}
Tenside sind Moleküle mit großer wissenschaftlicher und technologischer Bedeutung, da sie häufig in Reinigungsmitteln, als Emulgatoren und zur Herstellung diverser Nanostrukturen verwendet werden. Ihre faszinierenden Eigenschaften in Bezug auf selbstorganisierte Strukturen wie Mizellen oder Flüssigkristalle entstammen ihrem amphiphilen Grundgerüst - eine polare Kopfgruppe verknüpft mit einer hydrophoben Kette. Während die meisten der bekannten Tenside rein organisch sind, entwickelt sich gerade eine neue Tensidfamilie, welche den amphiphilen Charakter mit der erweiterten Funktionalität von Übergangsmetallen kombiniert, z. B. Redox- oder katalytische Eigenschaften sowie Magnetismus. Diese hybriden Tenside weisen aufgrund der einzigartigen Größe und der elektronischen Eigenschaften der metallhaltigen Teile auch neuartige Merkmale in der Selbstorganisation auf.
\end{abstract}

\section{Einleitung}

Tenside (SURFs; aus dem Englischen „surfactants“) stellen eine Unterklasse von großem Interesse der soge nannten amphiphilen Spezies dar. Sie zeichnen sich durch einen speziellen Aufbau mit Dipolcharakter aus, da zwei Bestandteile mit erheblich unterschiedlichen Lösungsmittel kompatibilitäten in einem Molekül vereint werden. Die ein fachsten Tenside bestehen aus einer hydrophilen Kopfgruppe, die an eine hydrophobe Alkylkette angebunden ist. Dieses einzigartige Merkmal ist verantwortlich für das bekannte Verhalten bezüglich Selbstorganisation und in zahlreichen Anwendungen. ${ }^{[1]}$ SURFs werden im Multitonnen Maßstab als Waschmittel, in Kosmetika, als Emulgatoren und als Phasentransferkatalysatoren eingesetzt. ${ }^{[2]}$ Die Nanowissen schaften hätten außerdem ohne Tenside nicht eine so rasante Entwicklung nehmen können, angesichts ihrer Fähigkeit, Phasen mit hohem Oberfläche zu Volumen Verhältnis wie Nanopartikel oder nanoporöse Materialien zu stabilisieren. ${ }^{[3]}$

Das Selbstorganisationsverhalten von SURFs ist kon zentrationsabhängig (Abbildung 1) und wurde umfangreich in Wasser untersucht: $\mathrm{Zu}$ Beginn besetzen einzelne Tensid moleküle die Grenzfläche zwischen Wasser und Luft, bis

[*] Prof. Dr. S. Polarz, Dr. S. Landsmann, A. Klaiber Fachbereich Chemie, Universität Konstanz 78457 Konstanz (Deutschland)

E Mail: sebastian.polarz@uni konstanz.de Homepage: http://cms.uni konstanz.de/polarz/

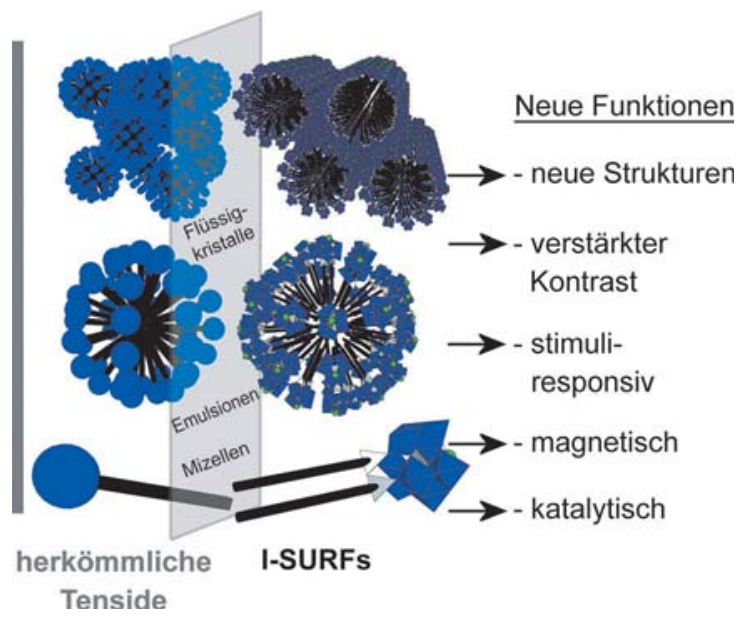

Abbildung 1. Ähnlichkeiten und Unterschiede zwischen anorganischen (I SURFs) und gewöhnlichen, rein organischen Tensiden.

diese dicht gepackt ist. Bei höherer Konzentration findet eine supramolekulare Strukturveränderung statt, und Mizellbil dung kann beobachtet werden. Die hydrophilen Kopfgruppen befinden sich in der Mizelle Wasser Grenzfläche, und die hydrophoben Reste zeigen nach innen.

Einige noch gültige Theorien zur Mizellbildung wurden von Israelachvili vor über 40 Jahren aufgestellt. ${ }^{[4]}$ Die Art der Aggregate, die bei mittleren Konzentrationen beobachtet werden, wird vom Packungsparameter bestimmt, einer cha 
rakteristischen Größe, die von der Form des einzelnen Ten sidmoleküls abhängt. Zum Beispiel haben Tenside mit langen Köpfen und kurzen Ketten einen kleinen Packungsparameter $(<1 / 3)$ und die Form eines Kegels. Deshalb tendieren diese Tenside dazu, Strukturen zu bilden, die sich durch eine starke Krümmung auszeichnen, wie Kugelmizellen. Bei höherer Konzentration kann man sogar die Bildung von Mesophasen oder lyotropen Flüssigkristallen (LLCs) induzieren. Bisher ist eine große Vielfalt von mehr oder weniger komplexen LLC Phasen bekannt, abhängig von der Komplexität der Tensid struktur. ${ }^{[4]}$

Praktisch alle Tenside, die in technologischen Zusam menhängen verwendet werden, sind organischer Natur mit Kopfgruppen wie Ammonium (kationisch), Oligoethylen glycol (neutral) oder Carboxylat (anionisch), um nur einige Beispiele zu nennen. Aus diesem Grund erscheint es sehr vielversprechend $\mathrm{zu}$ sein, Tenside mit den erweiterten Ei genschaften auszustatten, die charakteristisch für Über gangsmetallionen sind (z.B. verschiedene Redoxzustände, magnetisches Moment, katalytische Aktivität usw.; Abbil dung 1). Folglich können Tenside mit anorganischen Be standteilen (I SURFs) zur großen Klasse der Metallomeso gene gezählt werden. ${ }^{[5]}$ Der Begriff Metallomesogene wurde erstmals von Bruce et al. für molekulare Spezies verwendet, die flüssigkristalline (LC) Phasen bilden können, ausgehend von Komplexen als Bausteine. ${ }^{[6]}$ In den meisten seiner frühen Arbeiten konzentrierte er sich auf Systeme mit thermotropen LC Eigenschaften, zum Beispiel stäbchen oder plättchen förmige Metallkomponenten. ${ }^{[7]}$ Mittlerweile können auch einige metallhaltige Polymere und sogar anorganische Parti kel, die LC Phasen bilden, und manche Suprakristalle zu den Metallomesogenen gezählt werden. ${ }^{[8]}$ Es sollte ebenfalls er wähnt werden, dass metallgesteuerte Selbstanordnung eine große Rolle in heutigen Forschungsfeldern wie Koordinati onspolymere, Metall organische Gerüstverbindungen (MOFs) ${ }^{[9]}$ konjugierte metallhaltige Polymere ${ }^{[10]}$ oder $\mathrm{Me}$ tallodendrimere ${ }^{[11]}$ spielt.

Hier richten wir den Fokus auf die Herausstellung einiger der wichtigsten und jüngsten Entwicklungen auf dem Feld der Tensidsysteme mit anorganischen Bestandteilen. Besonders auf molekulare Systeme, die sich durch einen ausgeprägten Dipolcharakter auszeichnen und Selbstorganisationsphäno mene in Gegenwart von Lösungsmitteln zeigen (lyotropes Verhalten). Es gibt derzeit zwei Ansätze, um Tenside mit (Übergangs)Metalleinheiten auszustatten (Schema 1). Die einfachste Methode wird in Abschnitt 2 beschrieben. Sie verwendet konventionelle Spezies mit metallhaltigen Gege nionen (Typ I). Es ist jedoch sehr viel anspruchsvoller und erstrebenswerter, die Metalleinheit fest in das Tensid einzu binden (Typ II), was in Abschnitt 3 diskutiert wird.

\section{Tensidsysteme mit metallhaltigen Gegenionen (TYp I)}

Es ist offensichtlich, dass anionische Tenside mit Metall kationen als Gegenionen das denkbar einfachste I SURF System darstellen. ${ }^{[12]}$ Die ersten Beispiele für I SURFs (Typ I) wurden 1989 von Mirnaya et al. vorgestellt, als die
LC Bildung von Alkalimetall Alkylcarboxylat Schmelzen $\left(\mathrm{M}\left(\mathrm{OOCC}_{n} \mathrm{H}_{2 n+1}\right)\right)$ entdeckt wurden. ${ }^{[13]}$ Verwandte Systeme mit Erdalkalimetallen konnten durch die Verwendung orga nisch modifizierter Sulfonate erhalten werden. ${ }^{[14]}$ Aber auch kationische oder neutrale Tenside kombiniert mit Metallen

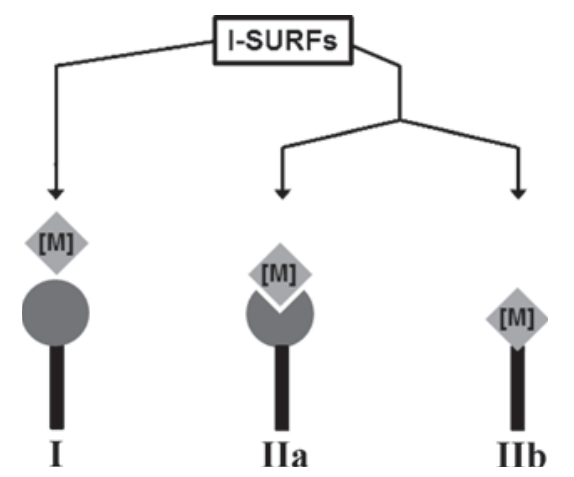

Schema 1. Klassifizierung verschiedener Typen von Tensiden mit anor ganischen Bestandteilen [M]. I) elektrostatische Wechselwirkung zwi schen Tensid und [M]; Ila) koordinative Wechselwirkung zwischen Kopfgruppe und [M]; IIb) kovalente Verknüpfung/[M] als Kopfgruppe. 
sind einen Blick wert. Die Wechselwirkung des kationischen SURF Cetyltrimethylammonium mit anionischen Silicatspe zies führte zur Entwicklung von geordneten mesoporösen Materialen über synergistische Coassoziation (,synergistic co assembly“). ${ }^{[15]}$ Mittlerweile sind zahlreiche Beispiele zur Herstellung von verschiedensten mesoporösen Metalloxid materialien mithilfe von Tensidphasen als Templaten be kannt. ${ }^{[3 a, 16]}$ Eine weitere interessante Idee ist es, anionische Metallspezies wie Palladate, Aluminate usw. mit kationischen Tensiden und im Besonderen mit ionischen Flüssigkeiten, ${ }^{[17]}$ also tensidähnliche molekulare Systeme mit großen kationi schen Kopfgruppen, zu kombinieren. Die meisten Beispiele hierzu konzentrierten sich auf katalytische Aktivität oder elektrochemische Anwendungen anstatt auf Selbstanord nung. ${ }^{[18]}$

Einige spektakuläre Ergebnisse, die besondere Auf merksamkeit verdienen, wurden durch die Verwendung von sogenannten Polyoxometallaten (POMs) als Gegenionen für kationische Ammoniumtenside erhalten. ${ }^{[19]}$ POMs bestehen aus mehrkernigen Metalloxideinheiten $\left[\mathrm{MO}_{x}\right]$ und sind be kannt für ihre interessanten katalytischen und magnetischen Eigenschaften. ${ }^{[20]}$ Die am besten untersuchte Klasse der POMs sind die Heteropolyanionen mit Keggin $\left(\left[\mathrm{XM}_{12} \mathrm{O}_{40}\right]^{n-}\right)$ oder Wells Dawson Struktur $\left(\left[\mathrm{X}_{2} \mathrm{M}_{18} \mathrm{O}_{62}\right]^{n-}\right)$ mit $\mathrm{M}=\mathrm{W}$, Mo und $\mathrm{X} \cong$ Zentralatom mit tetraedrischer Ko ordination, z. B. $\mathrm{P}^{\mathrm{V}}$. Komplexere Verbindungen können mit Isopolyanionen ohne Zentralatom erhalten werden. Müller et al. berichteten zum Beispiel in einer Reihe von bahnbre chenden Arbeiten, dass das Wachstum von nanoskaligen POM Clustern durch die partielle Reduktion, z. B. von $\mathrm{Mo}^{\mathrm{VI}}$ zu Mo ${ }^{\mathrm{V}}$ Spezies, erreicht werden kann. ${ }^{[21]}$ Aufgrund der rie sigen Zahl an Verbindungen, die zur Klasse der Polyoxome tallate gehören (vermutlich sind es einige Tausend), würde es den Rahmen dieses Kurzaufsatzes sprengen, einen ausgiebi gen Überblick über das POM Gebiet zu geben. ${ }^{[20 a]}$ Jedoch sind hybride POM SURF Systeme exzellente Beispiele für tensidverkapselte Cluster (,surfactant encapsulated clus ters", SECs). Kurth und Mitarbeiter erhielten SECs durch Ersetzen der Gegenionen anionischer Molybdovanadat POMs durch kationische Ammoniumtenside mit langen $\mathrm{Al}$ kylketten. ${ }^{[19 \mathrm{~b}, 22]}$ Hierbei wurde beobachtet, dass die Alkyl ketten eine dichte Packung einnehmen, was zu der Vermu tung führte, dass es eine kompakte Hülle um das POM gibt. Die Tensidhülle verbessert die Stabilität des umschlossenen POMs und dessen Löslichkeit in unpolaren, aprotischen or ganischen Lösungsmitteln. Darüber hinaus wurde das Selbstanordnungsverhalten genau untersucht. Durch die Langmuir Blodgett(LB) Methode ${ }^{[23]}$ abgeschiedene SECs bilden hochgeordnete und homogene Filme, welche nach Verdampfen des Lösungsmittels geordnete 2D Strukturen bilden.

Unsere Gruppe präsentierte 2001 ein einzigartiges Sys tem, das später von Cronin et al. „wiederentdeckt“ wur de. ${ }^{[19 e, 24]}$ Riesige, donutförmige POMs wurden mit kationi schen Tensiden mit je zwei Alkylketten umgesetzt. Es wurde festgestellt, dass das Tensid exklusiv an der Peripherie der anorganischen POM Cluster bindet (Abbildung 2) und da durch eine neue Spezies mit besonderen, quadrupolar am phiphilen Eigenschaften entsteht (hydrophiler harter anor ganischer Kern und hydrophobe weiche organische Hülle), welche dann eine kolumnare flüssigkristalline Phase bildet. Weiterhin wurde gezeigt, dass das anorganische Gerüst dem System zusätzliche katalytische Eigenschaften verleiht.

Eine wichtige Erweiterung des SEC Ansatzes wurde durch Kombination der Tensidverkapselung mit einer kova lenten Modifizierung des POM Clusters erreicht. Diese neu artigen Systeme sind unter tensidverkapselten, organisch modifizierten Polyoxometallaten („surfactant encapsulated organically grafted polyoxometalates“, SEOPs) bekannt, für die Wu et al. den Weg bereiteten. ${ }^{[25]}$ Sie statteten ein $\mathrm{Mn}$ Anderson POM mit stimuliresponsiven Einheiten wie Azo benzolgruppen aus, um intelligente selbstorganisierende Strukturen zu erhalten (Abbildung 3). Das Selbstanord nungsverhalten wird durch Lichtbestrahlung gesteuert, was zur Umbildung der Wasserstoffbrücken führt. UV Bestrah lung $(365 \mathrm{~nm})$ führt zur reversiblen Umwandlung der faseri gen Morphologie des SEOPs (mit Azobenzoleinheiten in trans Konfiguration) zu sphärischen Aggregaten. Das be schriebene ,intelligente“ Verhalten kann durch Variation der kovalent gebundenen organischen Reste des SEOPs verän dert werden. Entsprechend wurden ein SEOP mit thermo responsiven Eigenschaften für ein Adenin modifiziertes
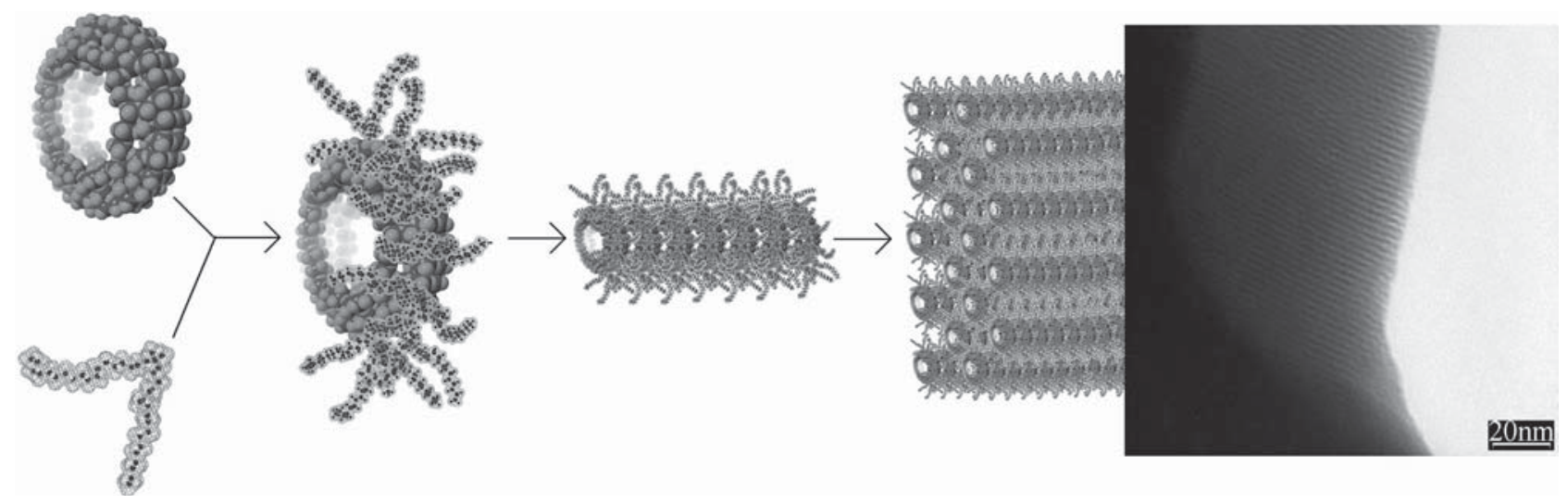

Abbildung 2. Selbstorganisation eines riesigen scheibenförmigen POMs (mit 176 Mo Atomen) durch elektrostatische Bindung von Dioctadecyl dimethylammonium als kationisches Tensid; Mechanismus und TEM Aufnahme. Wiedergabe mit Genehmigung nach Lit. [19e]. Copyright 2001, Wiley VCH. 


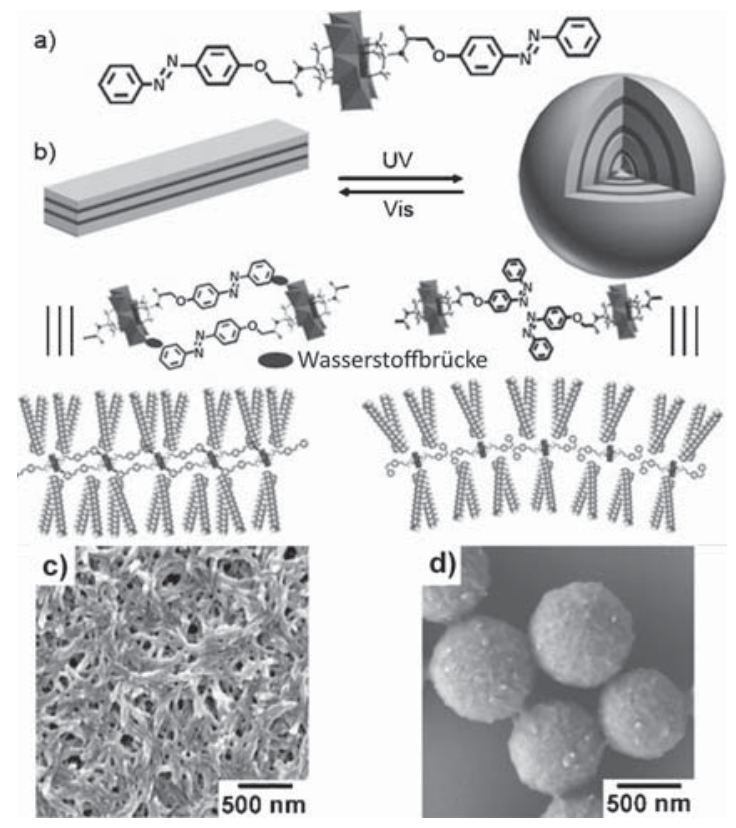

Abbildung 3. a) Struktur des mit Azobenzol modifizierten Mn Ander son POM Clusters. b) Schema der lichtinduzierten Selbstorganisation. SEM Bilder vor (c) und nach Bestrahlung (d). Wiedergabe mit Geneh migung nach Lit. [25]. Copyright 2010, Wiley VCH.

$\mathrm{POM}^{[26]}$ sowie ein SEOP, welches auf elektrische Reize rea giert, beschrieben, bei dem Ferrocen Einheiten verankert wurden. ${ }^{[27]}$ Ähnliches erwähnen einige neuere Arbeiten, die die Bildung interessanter Strukturen durch Reaktion von Tensiden mit metallhaltigen Polyelektrolyten oder Koordi nationspolymeren beschreiben. ${ }^{[28]}$

\section{Metalle als integraler Bestandteil der I-SURF- Kopfgruppe}

\subsection{I-SURFs mit organischen Liganden (TYp IIa)}

Der Zugang zu Tensiden mit einem Metallzentrum in der Kopfgruppe ist auf den ersten Blick sehr einfach. Die Grundidee ist, ein geladenes Metallkomplexfragment an ei nen organischen Chelatliganden zu koordinieren, der mit ei ner langen hydrophoben Kette modifiziert ist (Schema 2). Auf den zweiten Blick wird jedoch klar, dass dieser Ansatz

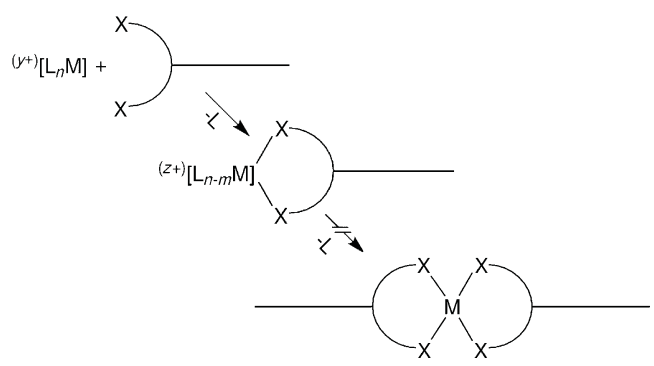

Schema 2. Typische Syntheseroute zu I SURFs mit organischen Ligan den, die lange Kohlenwasserstoffketten tragen ( $L$ Ligand; $M \quad M e$ tallzentrum; $X$ Donor). ziemlich anspruchsvoll ist. Schwierigkeiten bereitet die auf wendige Herstellung unsymmetrischer molekularer Spezies. Die Synthese der benötigten organischen Liganden kann sehr mühsam sein, und das Anbinden von nur einer Kohlenwas serstoffkette kann sich als schwierig erweisen. Darüber hinaus ist eine weitere Reaktion des Metallzentrums mit einem zweiten Liganden generell nicht erwünscht, da dies zu Sub stanzen mit geringerem dipolaren und erhöhtem hydropho ben Charakter führt (Schema 2). Ausnahmefälle treten auf, wenn die Koordinationsgeometrie des Metalls eine Orien tierung aller hydrophoben Seitenketten in eine Richtung er möglicht. ${ }^{[29]}$ Somit ist vorteilhaft, wenn das Metallzentrum eine niedrige Koordinationszahl bevorzugt oder der Ligand eine hohe Zähnigkeit aufweist.

Einige Beispiele, die immer noch eng mit I SURFs von Typ I verwandt sind, wurden von Bruce et al. vorgestellt. ${ }^{[30]}$ Sie geben an, dass der Austausch eines labilen Liganden in oktaedrisch koordinierten Komplexen gegen ein langkettiges Amin zu Tensidspezies führt; allerdings wurde die $\mathrm{M} \mathrm{N}$ Bindung in Wasser schnell gespalten. Le Moigne et al. haben komplexere Liganden verwendet, die Kronenether Einheiten zur Metallbindung mit dem Tensid Design verbanden ( $\mathrm{Ab}$ bildung 4 a). ${ }^{[31]}$ Ein ähnliches Tensidsystem und sein Selbst

a)

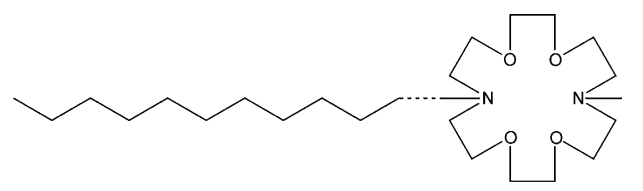

b)

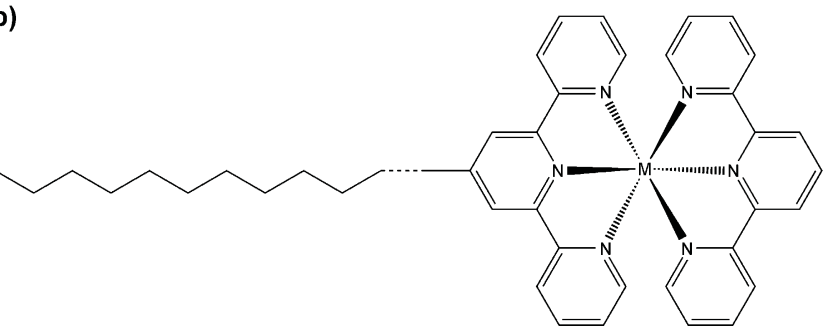

Abbildung 4. Zwei verschiedene Chelatliganden, verwendet für I SURFs vom Typ Ila. a) Tensid mit Kronenether Kopfgruppe (vor Metallkoordi nation). ${ }^{[3]]}$ b) I SURF mit Bipyridin Kopfgruppe. ${ }^{[33]}$

organisationsverhalten wurde von Neve et al. vorgestellt. ${ }^{[32]}$ Diese Veröffentlichung ist erwähnenswert, da die Autoren ihre $\mathrm{Ag}^{+}$haltigen Tenside erfolgreich durch eine Röntgen Einkristallstrukturanalyse charakterisieren konnten (Abbil dung 5), was nur sehr selten für SURF Systeme möglich ist.

Später führten Bruce und Mitarbeiter Bipyridinderivate und verwandte Substanzen als Liganden für die Synthese von I SURFs vom Typ IIa ein. ${ }^{[33,34]}$ Ein beeindruckendes Beispiel ist der in Abbildung $4 \mathrm{~b}$ gezeigte Bis(terpyridin) Komplex. ${ }^{[33]}$ Ein Tensid mit zwei Seitenketten und einem Vanadylkation als magnetisches Übergangsmetallzentrum wurde von Swager et al. präsentiert. ${ }^{[35]}$ Binnemans et al. tüftelten an Synthesen für lanthanoidhaltige Tenside. Sie stellten maßgeschneiderte siebenzähnige Liganden vor, die das Metallion umschlie Ben. ${ }^{[12 b, 36]}$ 


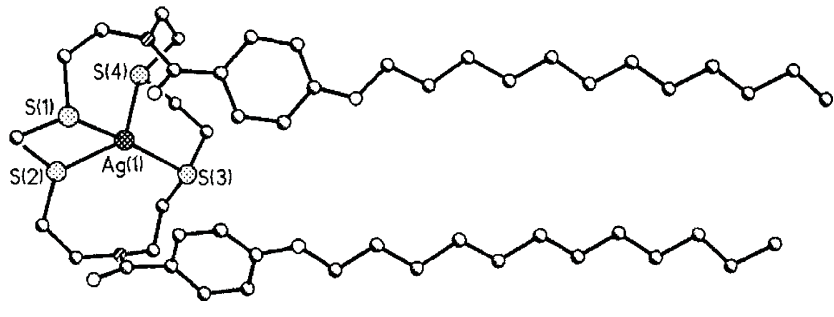

Abbildung 5. Struktur eines $\mathrm{Ag}^{+}$haltigen I SURF vom Typ Ila im Fest körper. Wiedergabe mit Genehmigung aus Lit. [29]. Copyright 1996, Wiley VCH.

Die meisten der genannten Veröffentlichungen konzen trieren sich auf die Synthese der Tensidmoleküle und ihre Untersuchung in Bezug auf Selbstorganisation (thermotrop und lyotrop). Eines der ersten Beispiele, das auf die erwei terten Eigenschaften von I SURFs hinweist, wurde in einer inspirierenden Arbeit von Matyjaszewski et al. präsentiert. ${ }^{[3]}$ Die Autoren stellten dreizähnige Stickstoffliganden her, die mit einer langen Alkylketten modifiziert wurden. Nach Ko ordination an $\mathrm{Cu}^{\mathrm{I}}$ war es möglich, dieses I SURF für die ra dikalische Atomtransferpolymerisation (ATRP) zu verwen den.

\subsection{I-SURFs mit anorganischen Resten als Kopfgruppe (TYp IIb)}

Tenside mit rein anorganischen Kopfgruppen (Schema 1; Typ IIb) sind viel seltener, und die ersten Beispiele wurden erst vor kurzem publiziert. Das Anbinden von mehrkernigen übergangsmetallhaltigen Resten an Alkylketten ist sehr schwierig aufgrund der Empfindlichkeit vieler M X Bindun gen $(\mathrm{X}=\mathrm{C}, \mathrm{O}, \mathrm{N})$ gegenüber Hydrolyse. Natürlich würde mit der Abspaltung der Alkylkette auch der amphiphile Cha rakter verschwinden. Allerdings lieferten aktuelle For schungsergebnisse auf dem Gebiet der Polyoxometallate (POMs) einige höchst innovative wasserstabile Tensidspezies, und es wurden einige interessante Studien zu deren Selbst anordnung veröffentlicht. ${ }^{[38]}$

Ein frühes amphiphiles POM System, ausgehend von ei nem Trisalkoxo modifizierten Mn Anderson POM, wurde von Cronin und Liu et al. vorgestellt. ${ }^{[39]}$ Eine Alkylkette wurde auf jeder Seite eines $\left[\mathrm{MnMo}_{6} \mathrm{O}_{24}\right]$ Clusters durch Amidbindungen angebracht. Es wurde gezeigt, dass sich das amphiphile System zu großen Vesikeln anordnet (Abbil dung 6). Dies war das erste Beispiel für eine vesikelartige Anordnung von POM Amphiphilen. Allerdings hat das ge nannte System zwei Nachteile: Das Amphiphil ist wegen der sperrigen TBA Gegenionen unlöslich in Wasser. Deshalb findet eine Selbstanordnung nur durch Zugabe signifikanter Mengen an Acetonitril statt. Darüber hinaus ist die Geome trie des Amphiphils ungünstig in Bezug auf den idealen Di polcharakter eines Tensids, da die hydrophoben Seitenketten symmetrisch auf beiden Seiten der Kopfgruppe angebracht sind. Als Konsequenz müssen die Ketten stark gebogen werden, um eine Vesikelstruktur zu bilden. Dies erschwert die Bildung des Vesikels, und der Selbstorganisationsprozess dauert im Vergleich zu klassischen Tensiden sehr lange

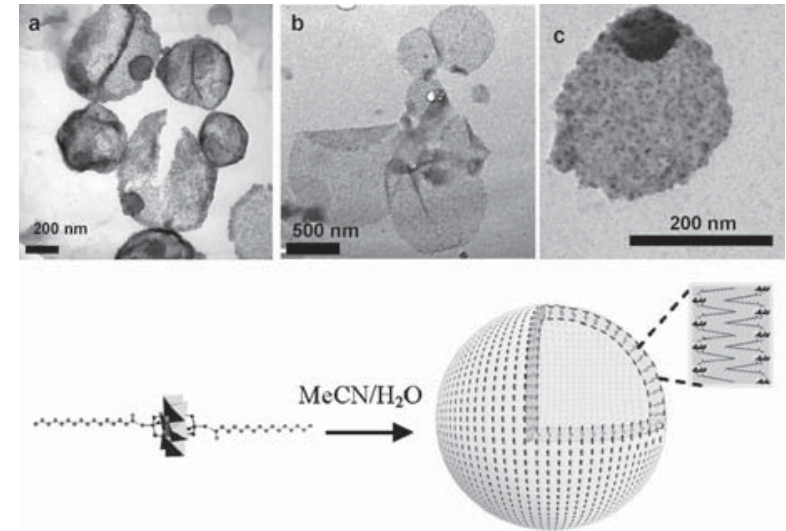

Abbildung 6. Vesikelbildung des Mn Anderson POM I SURF in einer Acetonitril Wasser Mischung; TEM Bilder und Bildungsmechanismus. Wiedergabe mit Genehmigung aus Lit. [39]. Copyright 2008, American Chemical Society.

(57 Tage um den Gleichgewichtszustand zu erreichen). Den noch ist es für ein Amphiphil mit dieser Geometrie günstiger, sich in apolaren Medien zu inversen Aggregaten anzuord nen. ${ }^{[40]}$ Diese inversen Vesikel mit relativ geringer Polydi spersität wurden auch gefunden. Die Länge des Alkylrests hat keinen großen Einfluss auf den Selbstanordnungsprozess. Jedoch verändert sich die Vesikelgröße je nach Polarität des Lösungsmittels.

Ein weiteres bemerkenswertes POM Tensid wurde von der Gruppe um Wang 2010 vorgestellt. Ein $V_{3}$ überdachtes Dawson POM wurde mit einer ATRP Initiatoreinheit aus gestattet und als Auslöser für eine Styrol Polymerisation verwendet. $^{[41]}$ Anschließend wurden die TBA Gegenionen durch Protonen ersetzt. Die DMF Lösung wurde trüb, was die zunehmende Amphiphilie des POM PS Bestandteils an zeigte. Die POM PS Amphiphile ordnen sich zu inversen Vesikeln mit einem mittleren Durchmesser von $166 \mathrm{~nm}$ an. Die Aggregate bestehen aus einer hellen Hülle, einem dunklen Kern und einem dünnen dunklen Ring zwischen dem Kern und der Hülle. Die Vesikel weisen eine Doppel schichtmembran auf, bei der die POM Cluster in der Mitte der Membran lokalisiert sind. Die berechnete Dicke der Membran beträgt $64 \mathrm{~nm}$, was ein riesiger Wert für Doppel schichtmembranen ist. ${ }^{[42]}$

Das besondere Anliegen unserer Gruppe ist es, POMs als integralen Bestandteil eines Tensids zu verwenden. Ein la kunares Wolframat vom Keggin Typ, $\left[\mathrm{PW}_{11} \mathrm{O}_{39}\right]^{7-}$, konnte durch Anbringen zweier langer Alkylketten modifiziert wer den (Abbildung 7). ${ }^{[43]}$ Dieses System erfüllt alle Schlüssel kriterien eines Amphiphils. Es kann verwendet werden, um Mizellen, Flüssigkristalle und sogar sehr stabile Emulsionen zu bilden. Allerdings wurde auch entdeckt, dass sich einige einzigartige Merkmale für das neue Tensidsystem abzeich nen, die über die bekannten Eigenschaften herkömmlicher organischer Tenside hinausgehen (Abbildung 1). Es gibt ei nen großen Unterschied in der Elektronendichte zwischen der Kopfgruppe mit 11 W Atomen und der Seitenkette $\left(\mathrm{CH}_{2}\right)_{n}(n=1220)$. Dies führt zu einem natürlichen Kontrast für zahlreiche Analysemethoden wie TEM und ebenfalls für Streumethoden. Die selbstangeordneten Strukturen können 


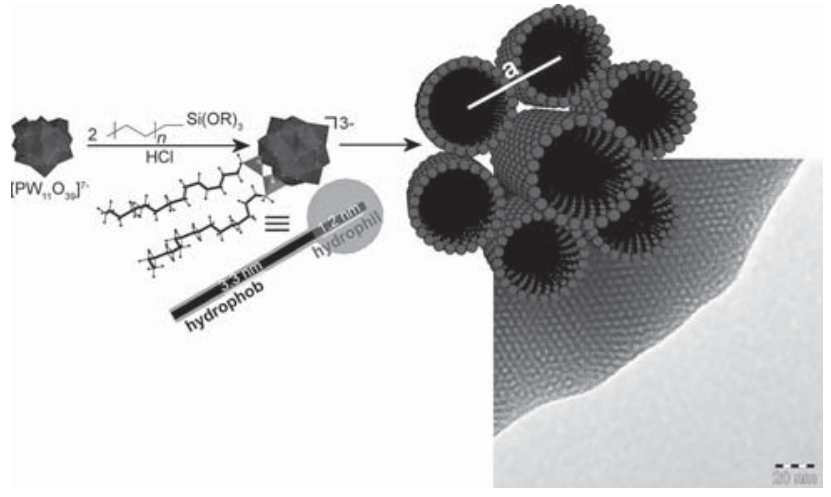

Abbildung 7. Synthese und LLC Bildung eines anionischen I SURFs mit einer $\left[\mathrm{PW}_{11} \mathrm{O}_{39}\right]^{3} \quad$ Kopfgruppe. Wiedergabe mit Genehmigung aus Lit. [43]. Copyright 2010, American Chemical Society.

somit präzise beobachtet werden. So konnte die Bildung einer Monoschicht an der Luft Wasser Grenzfläche durch GIXRD verfolgt werden. ${ }^{[44]}$ Die beobachteten supramolekularen 2D Strukturen, die sich an der Luft Wasser Grenzfläche bildeten, waren das erste Beispiel für die Aufklärung der Packung von hydrophilen Kopfgruppen und nicht von hydrophoben Sei tenketten. Deshalb stellen $\left[\mathrm{PW}_{11} \mathrm{O}_{39}\right]$ Verbindungen wert volle Modellsysteme in der Tensidforschung dar. ${ }^{[44]}$ Weiterhin konnten wir zeigen, dass das $\left[\mathrm{PW}_{11} \mathrm{O}_{39}\right]$ Tensid mit $\mathrm{H}^{+}$als Gegenion zwei Funktionen gleichzeitig erfüllen kann: Zum einen wirkt es als Emulgator, zum anderen als Polymerisati onskatalysator. ${ }^{[43,45]}$ Es ist offensichtlich, dass auch die restli chen Teile von POM Tensiden modifiziert werden können. Diese Teile sind das Gegenion der negativ geladenen Kopf gruppe, die hydrophobe(n) Kette(n) oder die Kopfgruppe selbst. Wir haben in einer neueren Publikation gezeigt, dass bereits die Modifikation der organischen Kette zu unerwar teten und neuen Tensideigenschaften führt. ${ }^{[46]}$ Es wurde unter anderem ein bipolares, Bola förmiges Tensid (Abbildung 8 a) hergestellt. Dieses Tensid bildet zum heutigen Zeitpunkt ei nes der kleinsten Monoschichtmembran(MLM) Vesikel in

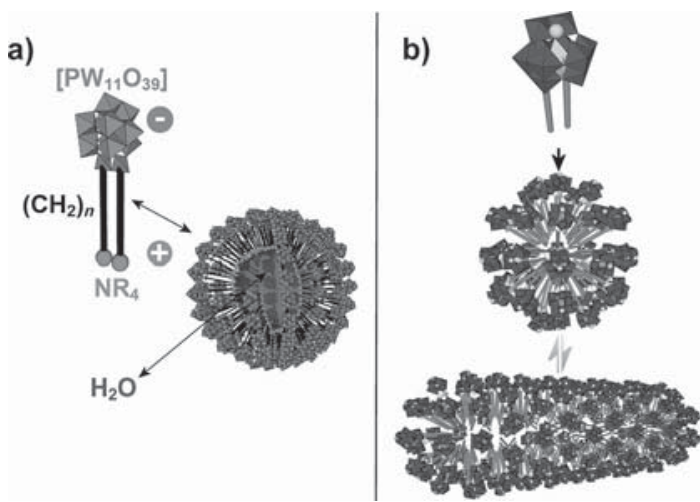

Abbildung 8. a) Ein bipolares $\left[\mathrm{PW}_{11} \mathrm{O}_{39}\right]$ Tensid und seine Organisation zu sehr kleinen Monoschichtvesikeln. Wiedergabe mit Genehmigung aus Lit. [46]. Copyright (2012) Nature Publishing. b) Mizellbildung von Dimetall POM I SURFs und das reversible elektrochemische Schalten von sphärischen zu stäbchenförmigen Mizellen. Wiedergabe mit Ge nehmigung aus Lit. [48]. Copyright 2012, Wiley VCH.
Wasser aus (Durchmesser $15 \mathrm{~nm}$ ), verbunden mit einer ex trem niedrigen kritischen Aggregationskonzentration (CAC) von nur $3 \times 10^{-6} \mathrm{molL}^{-1}$. Diese CAC liegt sogar unter den Werten für nichtionische polymere Detergenzien. ${ }^{[47]}$ Inzwi schen haben wir erste Schritte unternommen, das $\left[\mathrm{PW}_{11} \mathrm{O}_{39}\right]$ Tensidsystem weiter zu untersuchen. Die chemische Kom plexität der Kopfgruppe kann durch die Einführung eines zweiten Übergangsmetalls weiter gesteigert werden. ${ }^{[48]}$ Wir konnten zeigen, dass die Synthese einer Kopfgruppe mit Ruthenium als zweites Metall möglich ist. Über die typischen Tensideigenschaften hinaus werden infolge der speziellen anorganischen Kopfgruppe einige einzigartige Eigenschaften beobachtet: Im Unterschied zu bekannten Tensiden kann die Ladung der Kopfgruppe wegen der Redoxaktivität der ver knüpften Redoxsysteme $\mathrm{Ru}^{\mathrm{II} / I I / / \mathrm{V}}$ und $\mathrm{W}^{\mathrm{V} / \mathrm{VI}}$ reversibel gemäß $(1) \rightleftarrows(2) \rightleftarrows(3) \rightleftarrows(4)$ geschaltet werden. Zum ersten Mal war es möglich zu untersuchen, wie sich eine systematische Variation der Kopfgruppe auf das Selbstorganisationsverhal ten auswirkt. Wir zeigten durch Kombination von Analyse techniken wie Kleinwinkelröntgenstreuung (SAXS) und Ul trazentrifugation, dass eine Reduktion einen Übergang von gewöhnlichen sphärischen zu stäbchenförmigen Mizellen er gibt (Abbildung 8b). Eine weitere Konsequenz der be schriebenen Redoxchemie ist die Schaltbarkeit des Tensids von dia $\left(\mathrm{Ru}^{\mathrm{II}}\right) \mathrm{zu}$ paramagnetisch $\left(\mathrm{Ru}^{\mathrm{III}}\right)$, was durch EPR Spektroskopie nachgewiesen werden konnte. ${ }^{[48]}$

Später haben Liu und Wei ein System mit zwei Oktade cylketten präsentiert, die durch Esterbindungen auf zwei ge genüberliegenden Seiten eines Pentaerythritol modifizierten Hexavanadats mit Lindqvist Struktur verankert wurden. ${ }^{[49]}$ Es ist bemerkenswert, dass sich die Fluoreszenzeigenschaften dieses Amphiphils durch den Austausch des Gegenions des POMs von TBA zu H${ }^{+}$oder $\mathrm{Na}^{+}$ändert. Die ursprüngliche Emission entstammt wahrscheinlich einem Ligand zu Metall Charge Transfer Zustand. ${ }^{[50]}$ Darüber hinaus erhöht die Selbstanordnung des Amphiphils in Wasser die Fluoreszenz intensität. Dank der verbesserten Stabilität gegen Photo bleichen war es möglich, die selbstangeordneten Strukturen mithilfe von Fluoreszenzmikroskopie zu untersuchen. Wei terhin kann dieses System als hocheffizienter Emulsionska talysator für die Oxidation von Thiophen mit Wasserstoff peroxid verwendet werden. ${ }^{[51]}$ Es ist ebenfalls möglich, das Hexavanadat Ion mit alternativen, funktionelleren organi schen Gruppen zu modifizieren. Hill et al. beschrieben die Einführung von Pyreneinheiten über Amidbindungen ${ }^{\left[{ }^{[2]}\right.}$ Ihre Amphiphile ordnen sich zu vesikelartigen Strukturen an. Es wurde gezeigt, dass die Eigenschaften des Gegenions die su pramolekulare Packung der Vesikel beeinflussen: Je hydro phober das Gegenion, desto dichter ist die Packung der Vesikel. Das Fluoreszenzspektrum des I SURFs wurde dar aufhin von der Pyren Excimer Emission dominiert.

Cronin et al. entwickelten ein weiteres interessantes Bola förmiges POM I SURF System. ${ }^{[53]}$ Zwei $V_{3}$ überdachte Dawson POMs wurden mit verschiedenen organischen Lin kern verbunden. Das resultierende hantelförmige Molekül zeigt eine einzigartige Selbstorganisation zu MLM Vesikeln in Wasser Aceton Mischungen, was sehr unüblich für Bola förmige Tenside mit so geringem organischem Anteil ist. Normalerweise sind solch kurze hydrophobe organische 
Linker nicht in der Lage, den kompletten Raum zwischen den zwei hydrophilen Kopfgruppen zu füllen.

Die Gruppe um Liu erweiterte die Zahl hantelförmiger Systeme und studierte deren Selbstanordnungsverhalten ge nauer. ${ }^{[54]}$ Sie konnten die Größe eines Bola förmigen Tensids auf Basis eines Dawson POM durch Verwendung eines Bi pyridin Linkers auf $4.7 \mathrm{~nm}$ steigern. Es wurde weiterhin das Selbstanordnungsverhalten an der Wasser Luft Grenzfläche untersucht. Alle beschriebenen Bola förmigen Tenside bilden LB Monoschichten, jedoch hängt die Anordnung der Mo noschicht stark von der Hydrophobie des Linkers ab. Die stabilste LB Monoschicht wurde vom POM mit Bipyridin Linker gebildet, was mit einer zusätzlichen $\pi \pi$ Wechselwir kung der Bipyridine begründet wird, die diese Strukturen stabilisiert. ${ }^{[55]}$

Alle oben genannten Vorstufensysteme enthalten hydro phile Kopfgruppen kombiniert mit hydrophoben Alkylresten. Aus diesem Grund fragten wir uns, ob die Herstellung eines inversen I SURFs mit einem metallorganischen Oxocluster als Kopfgruppe möglich ist. Wir konnten erfolgreich eine Substanz herstellen, die einen Alkyl Alkoxy Zink Cluster trägt, verknüpft mit zwei Polyethylenglycol Ketten als polare Reste. (Abbildung 9) ${ }^{[56]}$ Interessanterweise zeigt dieses neue metallorganische Amphiphil Selbstanordnung über etliche Längenskalen. Zuerst bilden die Dimere lamellare Phasen durch Mikrophasentrennung der hydrophoben Oxocluster Einheiten und der polaren PEG Ketten aus. Dies wurde durch Polarisationsmikroskopie, SAXS und TEM nachge wiesen. ${ }^{[56]}$ In apolaren Lösungsmitteln wie Toluol kommt es zum Aufquellen der hydrophoben Domänen, und eine wei tere Strukturierung findet statt, überraschenderweise $\mathrm{zu} \mathrm{Ku}$ geln relativ einheitlicher Größe. Anschließend bilden diese Kugeln eine dichteste Packung, die sich aufgrund der Di mension der einzelnen Kugeln (200 nm Größe) wie ein opti sches Gitter verhält, das sichtbares Licht beugt. Dies führt zu einem blauen Farbeindruck (Abbildung 9), obwohl das Sys tem kein Chromophor enthält. Im Vergleich zu herkömmli chen Amphiphilen unterscheidet sich das beschriebene Sys tem auch durch eine zweite mögliche Funktion: die Ver wendung als $\mathrm{ZnO}$ Vorstufe. ${ }^{[56]}$ Es konnte tatsächlich bimo dales, makro und nanoporöses $\mathrm{ZnO}$ direkt aus nanostruk turiertem Gel hergestellt werden. Die Morphologie des resultierenden $\mathrm{ZnO}$ war direkt abhängig von der Morpholo gie der $[\mathrm{MeZnOPEG}]_{2}$ Phase.

\section{Schlussfolgerung}

Dieser Kurzaufsatz behandelt verschiedene Strategien, um Tenside mit metallhaltigen Kopfgruppen zu erhalten, und stellt einige der beeindrucktesten Literaturbeispiele vor. Es wurde gezeigt, dass es möglich ist, die klassischen Tensidei genschaften zu erweitern (Abbildung 1). Trotzdem kann die Synthese gewünschter I SURFs eine Herausforderung sein, und es gibt bisher nur wenige Beispiele. Ein besonderes Problem hierbei ist die Notwendigkeit, unsymmetrische Mo leküle herzustellen, bevorzugt mit ausgeprägt dipolarem Charakter. Die Besonderheit von I SURFs ist gegeben durch potenzielle synergistische Eigenschaften.

Synergie bedeutet, dass ein System mit zwei (oder mehr) Komponenten Fähigkeiten entwickelt, die keine der einzel nen Komponenten auszeichnen. Darüber hinaus sind die neuen Eigenschaften mehr als eine lineare Kombination, was sie sehr schwer vorhersehbar oder gar planbar macht. Des halb gibt es in der (Material)Chemie nur sehr wenige Bei spiele für Synergie. ${ }^{[57]}$ In diesem Zusammenhang ist es be merkenswert, dass die meisten der hier vorgestellten I SURFs einige absolut unerwartete Eigenschaften aufweisen, die als Ergebnis eines synergistischen Effektes interpretiert werden können. In den meisten vorgestellten Beispielen tre ten einzigartige Strukturen auf, die durch Selbstorganisation gebildet werden. Diese herausragende Eigenschaft von I SURF Systemen könnte zusätzlichen oder zumindest modi fizierten Wechselwirkungen zwischen Tensidmolekülen ge schuldet sein. Während für gewöhnliche Mesophasen Wech selwirkungen wie Dispersionskräfte, Wasserstoffbrücken, di polare Wechselwirkungen und der hydrophobe Effekt be rücksichtigt werden müssen, gibt es für I SURFs weitere mögliche Faktoren, z.B. magnetische Wechselwirkungen. Weiterhin kann nicht ausgeschlossen werden, dass die große

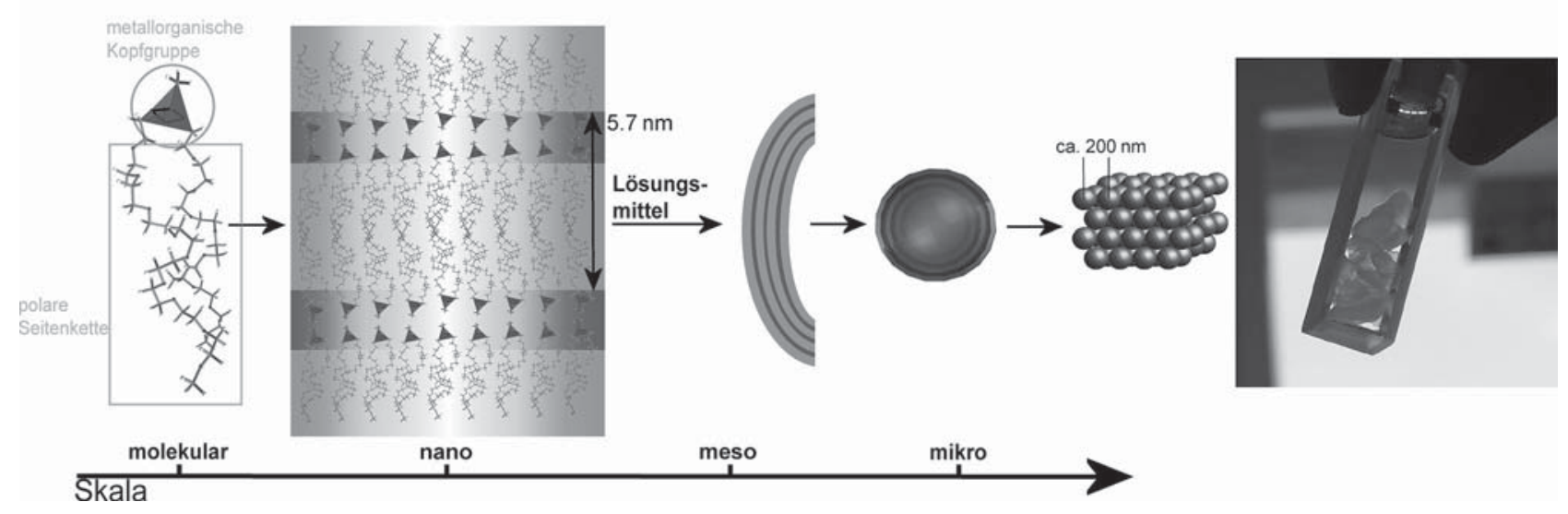

Abbildung 9. Struktur des ersten inversen metallorganischen I SURFs und dessen Selbstorganisation über mehrere Längenskalen. Rechts: Bilder des finalen Materials. Wiedergabe mit Genehmigung nach Lit. [56]. Copyright 2007, Wiley VCH. 
Ausdehnung der Kopfgruppe und eine eventuelle unsymme trische Verteilung der Ladungsdichte die Packung solcher hybriden Tenside beeinflusst.

Die Forschung auf dem Gebiet der anorganischen Tenside steht immer noch ganz am Anfang. Das Ziel zukünftiger Studien wird es sein, die Vielfalt der I SURFs zu erhöhen und ihre einzigartige Selbstorganisationseigenschaften zu verste hen. Darüber hinaus wird die Erforschung potenzieller An wendungsbereiche wie Katalyse, Wirkstofftransport oder in telligente Materialien neue Perspektiven in der Materialfor schung eröffnen.

[1] R. Nagarajan, E. Ruckenstein, Langmuir 1991, 7, 29342969.

[2] H. Stache, K. Kosswig, Surfactant Pocketbook, 3. Aufl., Hanser, München, 1990.

[3] a) S. Polarz, B. Smarsly, J. Nanosci. Nanotechnol. 2002, 2, 581 612; b) H. Weller, Curr. Opin. Colloid Interface Sci. 1998, 3, 194 199; c) S. Förster, T. Plantenberg, Angew. Chem. 2002, 114, 712 739; Angew. Chem. Int. Ed. 2002, 41, 689714.

[4] J. N. Israelachvili, Intermolecular and Surface Forces, Elsevier, Oxford, 2011.

[5] a) A. M. Giroud Godquin, P. M. Maitlis, Angew. Chem. 1991, 103, 370 398; Angew. Chem. Int. Ed. Engl. 1991, 30, 375 402; b) S. A. Hudson, P. M. Maitlis, Chem. Rev. 1993, 93, 861885.

[6] P. M. Maitlis, D. W. Bruce, R. Dhillon, D. A. Dunmur, F. P. Fa nizzi, S. E. Hunt, R. Lelagadec, E. Lalinde, R. Orr, J. P. Rourke, N. J. S. Salt, J. P. Stacey, P. Styring, New J. Chem. 1990, 14, 549 551.

[7] a) T. C. Bedard, J. S. Moore, J. Am. Chem. Soc. 1995, 117, 10662 10671; b) D. W. Bruce, Acc. Chem. Res. 2000, 33, 831840.

[8] a) L. Oriol, J. L. Serrano, Adv. Mater. 1995, 7, 348 369; b) X. H. Liu, D. W. Bruce, I. Manners, Chem. Commun. 1997, 289 290; c) G. R. Whittell, I. Manners, Adv. Mater. 2007, 19, 3439 3468; d) A. S. Sonin, J. Mater. Chem. 1998, 8, 2557 2574; e) A. L. Rogach, D. V. Talapin, E. V. Shevchenko, A. Kornowski, M. Haase, H. Weller, Adv. Funct. Mater. 2002, 12, 653 664; f) G. A Ozin, Chem. Commun. 2000, 419 432; g) S. Polarz, Adv. Funct. Mater. 2011, 21, 32143230.

[9] a) J. M. Lehn, Angew. Chem. 1988, 100, 91 116; Angew. Chem. Int. Ed. Engl. 1988, 27, 89 112; b) C. Janiak, Dalton Trans. 2003, 2781 2804; c) S. Kitagawa, R. Kitaura, S. Noro, Angew. Chem. 2004, 116, 2388 2430; Angew. Chem. Int. Ed. 2004, 43, 2334 2375.

[10] P. G. Pickup, J. Mater. Chem. 1999, 9, 16411653.

[11] a) E. C. Constable, Chem. Commun. 1997, 1073 1080; b) G. R. Newkome, E. F. He, C. N. Moorefield, Chem. Rev. 1999, 99, 16891746 .

[12] a) K. Binnemans, Chem. Rev. 2005, 105, 4148 4204; b) B. Donnio, Curr. Opin. Colloid Interface Sci. 2002, 7, 371394.

[13] T. A. Mirnaya, V. D. Prisyazhnyi, V. A. Shcherbakov, Usp. Khim. 1989, 58,14291450 .

[14] a) D. Tezak, F. Strajnar, O. Milat, M. Stubicar, Prog. Colloid Interface Sci. 1984, 69, 100 105; b) D. Tezak, O. Babacic, V. Derek, M. Galesic, S. Heimer, V. Hrust, Z. Ivezic, D. Jurkovic, S. Rupcic, V. Zelovic, Colloid Surf. A 1994, 90, 261 270; c) D. Tezak, M. Martinis, S. Puncec, I. Fischerpalkovic, F. Strajnar, Liq. Cryst. 1995, 19, 159167.

[15] a) Q. S. Huo, D. I. Margolese, U. Ciesla, P. Y. Feng, T. E. Gier, P. Sieger, R. Leon, P. M. Petroff, F. Schüth, G. D. Stucky, Nature 1994, 368, 317 321; b) A. Monnier, F. Schüth, Q. Huo, D. Ku mar, D. Margolese, R. S. Maxwell, G. D. Stucky, M. Krishnam urty, P. Petroff, A. Firouzi, M. Janicke, B. F. Chmelka, Science 1993, 261, 12991303.

[16] a) G. J. D. Soler illia, C. Sanchez, B. Lebeau, J. Patarin, Chem. Rev. 2002, 102, 4093 4138; b) P. D. Yang, D. Y. Zhao, D. I. Margolese, B. F. Chmelka, G. D. Stucky, Nature 1998, 396, 152 155.

[17] a) T. Welton, Chem. Rev. 1999, 99, 2071 2083; b) J. Dupont, R. F. de Souza, P. A. Z. Suarez, Chem. Rev. 2002, 102, 3667 3691.

[18] a) P. Wasserscheid, W. Keim, Angew. Chem. 2000, 112, 3926 3945; Angew. Chem. Int. Ed. 2000, 39, 3772 3789; b) T. Welton, Coord. Chem. Rev. 2004, 248, 2459 2477; c) M. Armand, F. Endres, D. R. MacFarlane, H. Ohno, B. Scrosati, Nat. Mater. 2009, 8, 621629 .

[19] a) E. Coronado, C. J. Gomez Garcia, Chem. Rev. 1998, 98, 273 296; b) D. G. Kurth, P. Lehmann, D. Volkmer, H. Cölfen, M. J. Koop, A. Müller, A. Du Chesne, Chem. Eur. J. 2000, 6, 385 393; c) H. Li, H. Sun, W. Qi, M. Xu, L. Wu, Angew. Chem. 2007, 119, 1322 1325; Angew. Chem. Int. Ed. 2007, 46, 1300 1303; d) A. Nisar, X. Wang, Dalton Trans. 2012, 41, 9832 9845; e) S. Polarz, B. Smarsly, M. Antonietti, ChemPhysChem 2001, 2, 457 461; f) W. Bu, S. Uchida, N. Mizuno, Angew. Chem. 2009, 121, 8431 8434; Angew. Chem. Int. Ed. 2009, 48, 82818284.

[20] a) D. L. Long, E. Burkholder, L. Cronin, Chem. Soc. Rev. 2007, 36, 105 121; b) A. Proust, R. Thouvenot, P. Gouzerh, Chem. Commun. 2008, 18371852.

[21] a) U. Kortz, A. Müller, J. van Slageren, J. Schnack, N. S. Dalal, M. Dressel, Coord. Chem. Rev. 2009, 253, 2315 2327; b) A. Müller, E. Beckmann, H. Bogge, M. Schmidtmann, A. Dress, Angew. Chem. 2002, 114, 1210; Angew. Chem. Int. Ed. 2002, 41, 1162.

[22] F. Caruso, D. G. Kurth, D. Volkmer, M. J. Koop, A. Müller, Langmuir 1998, 14, 34623465.

[23] A. Ulman, Chem. Rev. 1996, 96, 15331554.

[24] S. i. Noro, R. Tsunashima, Y. Kamiya, K. Uemura, H. Kita, L. Cronin, T. Akutagawa, T. Nakamura, Angew. Chem. 2009, 121, 8859 8862; Angew. Chem. Int. Ed. 2009, 48, 87038706.

[25] Y. Yan, H. Wang, B. Li, G. Hou, Z. Yin, L. Wu, V. W. Yam, Angew. Chem. 2010, 122, 9419 9422; Angew. Chem. Int. Ed. 2010, 49, 92339236.

[26] Z. He, Y. Yan, B. Li, H. Ai, H. Wang, H. Li, L. Wu, Dalton Trans. 2012, 41, 1004310051.

[27] Y. Yan, B. Li, Q. He, Z. He, H. Ai, H. Wang, Z. Yin, L. Wu, Soft Matter 2012, 8, 1593.

[28] a) G. Schwarz, Y. Bodenthin, Z. Tomkowicz, W. Haase, T. Geue, J. Kohlbrecher, U. Pietsch, D. G. Kurth, J. Am. Chem. Soc. 2011, 133, 547558 ; b) S. C. Junggeburth, L. Diehl, S. Werner, V. Duppel, W. Sigle, B. V. Lotsch, J. Am. Chem. Soc. 2013, 135, 61576164.

[29] F. Neve, Adv. Mater. 1996, 8, 277289.

[30] a) D. W. Bruce, D. A. Dunmur, P. M. Maitlis, J. M. Watkins, G. J. T. Tiddy, Liq. Cryst. 1992, 11, 127 133; b) D. W. Bruce, I. R. Denby, G. J. T. Tiddy, J. M. Watkins, J. Mater. Chem. 1993, 3, 911916.

[31] J. Le Moigne, J. Simon, J. Phys. Chem. 1980, 84, 170177.

[32] F. Neve, M. Ghedini, G. Demunno, A. M. Levelut, Chem. Mater. 1995, 7, 688693.

[33] J. D. Holbrey, G. J. T. Tiddy, D. W. Bruce, Dalton Trans. 1995, 17691774.

[34] D. W. Bruce, J. D. Holbrey, A. R. Tajbakhsh, G. J. T. Tiddy, J. Mater. Chem. 1993, 3, 905906.

[35] S. S. Zhu, T. M. Swager, Adv. Mater. 1995, 7, 280283.

[36] K. Binnemans, C. Gorller Walrand, Chem. Rev. 2002, 102, 2303 2345.

[37] K. Matyjaszewski, B. Göbelt, H. j. Paik, C. P. Horwitz, Ma cromolecules 2001, 34, 430440. 
[38] a) M. H. Rosnes, C. Musumeci, C. P. Pradeep, J. S. Mathieson, D. L. Long, Y. F. Song, B. Pignataro, R. Cogdell, L. Cronin, $J$. Am. Chem. Soc. 2010, 132, 15490 15492; b) D. L. Long, R. Tsunashima, L. Cronin, Angew. Chem. 2010, 122, 1780 1803; Angew. Chem. Int. Ed. 2010, 49, 1736 1758; c) A. Dolbecq, E. Dumas, C. R. Mayer, P. Mialane, Chem. Rev. 2010, 110, 6009 6048; d) A. Proust, B. Matt, R. Villanneau, G. Guillemot, P. Gouzerh, G. Izzet, Chem. Soc. Rev. 2012, 41, 7605 7622; e) C. Sanchez, G. J. A. A. Soler Illia, F. Ribot, T. Lalot, C. R. Mayer, V. Cabuil, Chem. Mater. 2001, 13, 3061 3083; f) P. Yin, L. Jin, D. Li, P. Cheng, D. V. Vezenov, E. Bitterlich, X. Wu, Z. Peng, T. Liu, Chemistry 2012, 18, 6754 6758; g) Y. F. Song, R. Tsunashima, Chem. Soc. Rev. 2012, 41, 7384 7402; h) P. Yin, D. Li, T. Liu, Chem. Soc. Rev. 2012, 41, 73687383.

[39] J. Zhang, Y. F. Song, L. Cronin, T. Liu, J. Am. Chem. Soc. 2008, 130, 1440814409.

[40] J. Zhang, Y. F. Song, L. Cronin, T. Liu, Chemistry 2010, 16, 1132011324.

[41] Y. Han, Y. Xiao, Z. Zhang, B. Liu, P. Zheng, S. He, W. Wang, Macromolecules 2009, 42, 65436548.

[42] Y. Xiao, Y. K. Han, N. Xia, M. B. Hu, P. Zheng, W. Wang, Chemistry 2012, 18, 1132511333.

[43] S. Landsmann, C. Lizandara Pueyo, S. Polarz, J. Am. Chem. Soc. 2010, 132, 53155321

[44] J. J. Giner Casares, G. Brezesinski, H. Mohwald, S. Landsmann, S. Polarz, J. Phys. Chem. Lett. 2012, 3, 322326.

[45] S. Polarz, S. Landsmann, Z. Anorg. Allg. Chem. 2010, 636, 2038.

[46] S. Landsmann, M. Luka, S. Polarz, Nat. Commun. 2012, 3, 1299.
[47] D. Schwarz, F. Junge, F. Durst, N. Frölich, B. Schneider, S. Re ckel, S. Sobhanifar, V. Dötsch, F. Bernhard, Nat. Protoc. 2007, 2, 29452957.

[48] S. Landsmann, M. Wessig, M. Schmid, H. Colfen, S. Polarz, Angew. Chem. 2012, 124, 6097 6101; Angew. Chem. Int. Ed. 2012, 51, 59955999.

[49] P. Yin, P. Wu, Z. Xiao, D. Li, E. Bitterlich, J. Zhang, P. Cheng, D. V. Vezenov, T. Liu, Y. Wei, Angew. Chem. 2011, 123, 2569 2573; Angew. Chem. Int. Ed. 2011, 50, 25212525.

[50] L. Chen, F. Jiang, Z. Lin, Y. Zhou, C. Yue, M. Hong, J. Am. Chem. Soc. 2005, 127, 85888589.

[51] P. Yin, J. Wang, Z. Xiao, P. Wu, Y. Wei, T. Liu, Chem. Eur. J. 2012, $18,91749178$.

[52] D. Li, J. Song, P. Yin, S. Simotwo, A. J. Bassler, Y. Aung, J. E. Roberts, K. I. Hardcastle, C. L. Hill, T. Liu, J. Am. Chem. Soc. 2011, 133, 1401014016.

[53] C. P. Pradeep, M. F. Misdrahi, F. Y. Li, J. Zhang, L. Xu, D. L. Long, T. Liu, L. Cronin, Angew. Chem. 2009, 121, 84598463 ; Angew. Chem. Int. Ed. 2009, 48, 83098313.

[54] M. F. Misdrahi, M. Wang, C. P. Pradeep, F. Y. Li, C. Lydon, L. Xu, L. Cronin, T. Liu, Langmuir 2011, 27, 91939202.

[55] V. M. Kaganer, H. Möhwald, P. Dutta, Rev. Mod. Phys. 1999, 71, 779819.

[56] S. Polarz, R. Regenspurger, J. Hartmann, Angew. Chem. 2007, 119, 2478 2482; Angew. Chem. Int. Ed. 2007, 46, 24262430.

[57] a) J. J. Becker, M. R. Gagne, Acc. Chem. Res. 2004, 37, 798 804; b) P. Gomez Romero, Adv. Mater. 2001, 13, 163174. 